\section{OP0259 ZCCHC5, A LTR RETROTRANSPOSON-DERIVED NEOFUNCTIONALIZED GENE, IS ESSENTIAL FOR THE TRANSCRIPTIONAL ACTIVITY OF SOX9 AND THE EXPRESSION OF COL2A1 IN CHONDROCYTES AND IS DOWNREGULATED IN OA CARTILAGE}

T.M. Haqqi, N.M. Khan. Anatomy and Neurobiology, North East Ohio Medical University, Rootstown, USA

Background: Retrotransposon-derived DNA sequences occupy approximately $40 \%$ of the mammalian genome, compared with only $1.5 \%$ of protein coding genes, and are a source of variation in the genome. LTR-derived gene ZCCHC5 (Mart3) is a member of gag-related retrotransposon family that has lost the ability to retrotranspose. $\mathrm{ZCCHC5}$ gene encode a protein of approximately $53 \mathrm{Kd}$ and contains a nucleic acid binding domain $\left(\mathrm{CX}_{2} \mathrm{CX}_{4} \mathrm{HX}_{4} \mathrm{C}\right)$, a gag-like region within the intact ORF and a homeobox associated leucine zipper motif indicating that this gene may have acquired new function(s) in the cell. Expression and function of $Z C C H C 5$ in degenerative joint diseases such as OA or other diseases has not been explored.

Objectives: The aim of this study was to investigate whether ZCCHC5 is expressed in $\mathrm{OA}$ cartilage and chondrocytes and whether it is involved in the regulation of catabolic and/or anabolic factors in chondrocytes and its modulation under pathological conditions.

Methods: Chondrocytes were derived by the enzymatic digestion of human OA and normal C57BL6 mouse cartilage. Total RNA was prepared using Trizol and was made DNA-free using on-column digestion. mRNA expression was quantified using TaqMan assays. Protein expression was determined by immunohistochemistry $(\mathrm{IHC})$ and Western blotting (WB) with validated antibodies. siRNA mediated depletion or plasmid mediated overexpression of ZCCHC5 gene was used to study its role in chondrocyte function under pathological conditions. Luciferase reporter vectors were used to study promoter activity in human chondrocytes.

Results: TaqMan, WB and $\mathrm{IHC}$ analyses revealed that the expression of ZCCHC5 was very low in the damaged OA cartilage compared to the levels detected in normal cartilage and the unaffected cartilage of the same patient. Stimulation of human and mouse chondrocytes with IL-1 $\beta$ significantly decreased the expression of ZCCHC5 $(p<0.05)$ which correlated with the inhibition of COL2A1 expression and upregulation of MMP-13. Overexpression of ZCCHC5 upregulated the COL2A1 mRNA and protein levels which were not suppressed by stimulation with IL-1 $\beta$. Importantly, in these cells expression of MMP-13 was low and remained suppressed under pathological conditions. In contrast siRNA mediated depletion of $\mathrm{ZCCHC} 5$ expression in chondrocytes abrogated the protective effect on COL2A1 expression and increased the level of MMP-13 mRNA and protein upon treatment with IL-1b. Additionally, depletion of ZCCHC5 eliminated the SOX9 activity and COL2A1 promoter activation in human chondrocytes but this was reversed by reintroduction of ZCCHC5 expression indicating that ZCCHC5 plays an important role in SOX 9 activity and COL2A1 transcription.

Conclusions: Our data for the first time demonstrate that a LTR retrotransposonderived neogene $\mathrm{ZCCHC} 5$ plays a key role in maintaining the differentiated functions of adult articular chondrocytes. Its repression in OA may contribute to cartilage deterioration by blocking chondrocyte anabolic functions and by enhancing expression of catabolic factors. Taken together, our data revealed a previously unidentified role of ZCCHC5 in a disease pathogenesis and uncovered a potential therapeutic approach to limit/reverse cartilage damage in OA.

Acknowledgements: Supported by NIH grants RO1 AT-007373, RO1 AR067056 and funds from the Northeast Ohio Medical University

Disclosure of Interest: None declared

DOI: 10.1136/annrheumdis-2018-eular.5943

\section{OP0260 TARGETING NEUTROPHIL MICROVESICLES TO DAMAGED CARTILAGE USING ANTIBODIES TO POST- TRANSLATIONALLY MODIFIED COLLAGEN II}

L.M. Topping, C. Vinci, H. Rhys, L. Norling, A. Nissim. WHRI, Queen Mary University of London, London, UK

Background: Microvesicles (MV) are extracellular vesicles released from the plasma membrane of cells. MV derived from neutrophils (PMN) have been shown to penetrate cartilage and exert chondro-protective effects in inflammatory arthritis

Collagen type II (CII) is the most abundant protein found in cartilage. Inflammation in the joint results in post-translational modification of collagen II (CII) by reactive oxygen species (ROS). We have produced a single chain variable fragment (scFv) antibody specific to Cll modified by ROS, namely anti-ROS-CII ${ }^{3}$. We hypothesise that loading anti-ROS-CII upon MV will localise them to the arthritic cartilage.

Objectives: The main objectives of this study were: i) Load anti-ROS-CII upon MV using aqueous energy dissemination; ii) Assess incorporation of anti-ROS-CII upon MV in vitro and iii) Validate the localisation and therapeutic potential of the enriched microvesicles in an in vivo model of arthritis.
Methods: Anti-ROS-CII were produced in house. Human PMN MV from healthy donors were isolated as previously described ${ }^{4}$. Anti-ROS-CII were intercalated upon MV using 100 ug 1,2-dioleoyl-sn-glycero-3-phospho-l-serine by aqueous energy dissemination, as previously described ${ }^{4-5}$. Incorporation was assessed by Imagestream ${ }^{\times}\left({ }^{\times}{ }^{\times}\right)$, ELISA, NanoSight and immunofluorescence. In vivo experiments were performed in an Antigen Induced Arthritis (AIA) mouse model. Localisationwas assessed by IVIS Imaging following intravenous treatment of enriched MV. Ex vivo IVIS imaging was performed to confirm in vivo data. In vivo experiments were also conducted using MV enriched with anti-ROS-CII fused to vlL-10 and anti-mTNF therapies. Clinical scores were assessed throughout. Knee joints were harvested and snap frozen to detect the presence of enriched MV in the arthritic cartilage by confocal microscopy, and for qPCR analysis.

Results: Incorporation of anti-ROS-CII upon MV was observed by Imagestream. Antibodies present on the MV retained their binding capabilities, as shown by ELISA. Strong microvesicle fluorescence was detected in the arthritic cartilage of mouse tissue sections when using anti-ROS-Cll enriched MV as a primary incubation.

In vivo studies exhibited the ability of the enriched MV to localise specifically in the arthritic joint. Ex vivo IVIS knee imaging confirmed in vivo results. Ex vivo knee cryosections showed the presence of enriched MV specifically within the arthritic cartilage.

In vivo treatment studies using anti-ROS-CII fused to anti-inflammatory therapeutics led to a significant reduction in knee swelling.
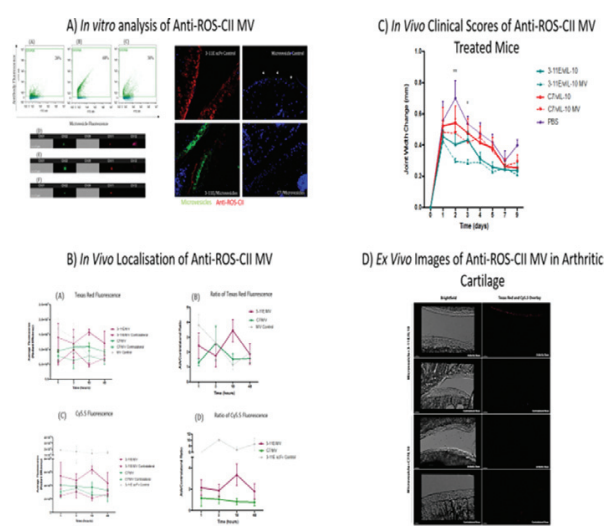

Conclusions: In this study we have demonstrated the ability to use aqueous energy dissemination to successfully enrich MV with antibodies. These enriched MV are able to localise in the arthritic joint and deliver anti-inflammatory therapeutics.

Overall, this study demonstrates the attainability of targeting a biological scaffold to the arthritic joint. The potential of co-delivering MV alongside anti-inflammatory therapeutics is paramount to simultaneously protect cartilage and reduce inflammation.

\section{REFERENCES}

[1] Dalli J, et al. Blood 2008;112:2512-9.

[2] Headland SE, et al. Science Translational Medicine 2015;7(315).

[3] Hughes C, et al. Arthritis and Rheumatism 2010;62(4):1007-1016.

[4] Norling LV, et al. The Journal of Immunology 2011;186(10):5543-5547

[5] Dalli J, et al. EMBO Molecular Medicine 2014;6(1):27-42.

Disclosure of Interest: None declared

DOI: 10.1136/annrheumdis-2018-eular.6744

\section{OP0261 \\ RETINOIC ACID IS REGULATED BY CARTILAGE INJURY AND IS ANTI-INFLAMMATORY IN HAND OSTEOARTHRITIS}

L. Zhu, H. Ismail, A. Chanalaris, M. Gardiner, T.L. Vincent, on behalf of ECHO. Kennedy Institute of Rheumatology, Oxford, UK

Background: A Genome-Wide Association Study (GWAS) in hand OA has identified the association of hypomorphic variants within ALDH1A2 and severe hand OA (Styrkarsdottir U, et al. 2014). This gene encodes the enzyme catalysing the production of all-trans retinoic acid (atRA). atRA has an essential role in embryonic limb development, but its role in adult cartilage and in OA remains unclear Previous work from our lab has shown that cartilage injury activates inflammatory signalling and regulates the expression of inflammatory genes. 
Trapeziectomy is a surgical treatment for patients with intractable base of thumb $O A$ and is a good source of hand OA cartilage.

Objectives: The aim of this study was to investigate the expression of atRAdependent and inflammatory genes in the cartilage of patients and to examine these with respect to the presence/absence of polymorphic ALDH1A2 variants. We also investigate the regulation of atRA upon cartilage injury and its effect on injury-induced inflammatory gene regulation.

Methods: We collected 26 trapeziectomy samples, and dissected the cartilage within one hour of collection. Genomic DNA was extracted for the identification of the two common variants (SNP rs4238326 and SNP rs3204689). Expression levels of atRA-dependent and inflammatory genes were tested by RT-PCR.

Healthy cartilage was obtained from femoral heads of 5-week-old mice and porcine metacarpophalangeal joints of 3-6 months old pigs. Gene regulation upon cartilage injury was tested. To examine the role of specific injury-induced pathways, porcine joints were pre-injected with selective inhibitors to: TGF $\beta$, FGF2R, TAK1 and CYP26, 1 hour prior to injury.

Results: Polymorphic variants in ALDH1A2 were common in this patient population and we identified 8 patients homozygous for both variants, and 5 patients who were wild type for both variants. mRNA levels of ALDH1A2 and atRA-dependent gene CYP19A were significantly lower in the homozygous group compared to wild type (figure 1). There were also trends in the regulation of several other atRAdependent genes. Conversely, inflammatory genes such as HAS1, TSG6 and ADAMTS5 showed a general increase in homozygous patients.

Cartilage injury in both porcine and murine tissue led to a rapid down-regulation of atRA-dependent genes (CYP26s and RARs). Injecting joints with a potent TAK1 inhibitor prevented the drop of atRA-dependent genes. Prior injection of the joint with a CYP26 (enzymes that normally break down atRA) inhibitor, restored levels of atRA-dependent genes after injury and suppressed injury-induced inflammation genes.

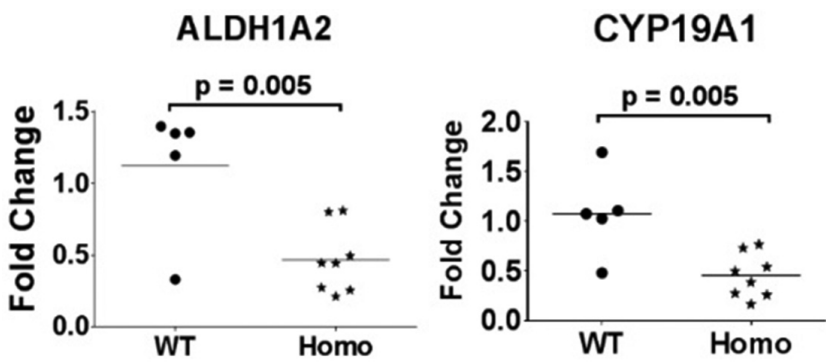

Conclusions: Polymorphic variants in ALDH1A2 are associated with significantly lower levels of ALDH1A2 and CYP19A1 mRNA in hand OA cartilage. Adult articular cartilage constitutively produces atRA, and this is strongly suppressed by mechanical injury through TAK1 activation. Preventing the drop in cellular atRA upon injury, by pre-incubating the joint with a CYP26 inhibitor, restores atRA levels and leads to a reduction in inflammatory gene regulation. These results indicate that atRA plays an important anti-inflammatory role in cartilage and provides a potential novel therapeutic strategy to treat hand $O A$

Disclosure of Interest: None declared

DOI: 10.1136/annrheumdis-2018-eular.3647

\section{OP0262 \\ DISEASE MODIFYING EFFECTS OF THE CANINE IL4-10 FUSION PROTEIN IN THE CANINE GROOVE MODEL OF OSTEOARTHRITIS}

E.M. Van Helvoort ${ }^{1}$, J. Popov-Celeketic ${ }^{1}$, K. Coeleveld ${ }^{1}$, A. Doornenbal ${ }^{2}$, M. Tryfonidou ${ }^{2}$, C. Wijne ${ }^{1}$, F. Lafeber ${ }^{1}$, S. Mastbergen ${ }^{1} .{ }^{1}$ Rheumatology and Clinical Immunology, University Medical Centre Utrecht, Utrecht University; ${ }^{2}$ Department of Clinical Sciences of Companion Animals, Faculty of Veterinary Medicine, Utrecht University, Utrecht, Netherlands

Background: An ideal disease modifying osteoarthritis (OA) drug should have analgesic, chondroprotective and anti-inflammatory effects. A fusion protein of Interleukin 4 and 10 (IL4-10FP) might have these effects.

Objectives: This study evaluates the effects of canine IL4-10FP (cIL4-10FP) in the canine Groove model of OA.

Methods: In 8 skeletally mature dogs, knee OA in the right leg was induced according to the Groove model. After 6 weeks of OA development, intra-articular injections in the affected knee with either PBS (500 $\mu$; n=4) or clL4-10FP (10 $\mu \mathrm{g} /$ $500 \mu \mathrm{l} ; \mathrm{n}=4)$ were given weekly for 10 weeks. The contra-lateral joints served as a healthy control.

Force plate analysis (FPA) was used to determine joint loading as a surrogate marker of pain. The ratio of the affected over the contra-lateral control joint was calculated for each dog for each time point. FPA was performed before OA induction to determine baseline values, and before and 24 hours after the 1 st, 6 th and 9th intra-articular injection. A linear mixed model was used to evaluate effects of injections. After 10 weeks of treatment dogs were euthanized and tissue samples were harvested. Serum Immunoglobulin G (IgGs) titers against clL4-10FP were evaluated to check for potential antibody formation.

Cartilage proteoglycan content and release of proteoglycans were determined ex vivo by Alcian Blue assay. Synovial inflammation was evaluated by HE-staining using OARSI grading. Changes in outcomes in the affected/treated joints compared to contra-lateral control joints were calculated.

Results: After OA induction a clear reduction in joint loading (increase in pain) was found (standing force and braking force). After clL4-10FP injections these forces increased toward normalisation (clL4-10FP vs PBS group, $p=0.002$ and $p=0.01$, respectively). No IgG elevation was detected after 10 injections. Compared to contra-lateral controls, proteoglycan content of OA PBS injected knees suggested tissue degeneration (27 vs $34 \mathrm{mg} / \mathrm{g}$ ). In the clL4-10FP treated group proteoglycan content in right knees was not different from the contralateral controls ( 33 vs $31 \mathrm{mg} / \mathrm{g}$ ). The mean change in proteoglycan content compared to contra-lateral controls was different with a $p$ value of 0.057 (figure 1). A similar pattern was found for the change in release of proteoglycans from the cartilage, which was less increased in the cIL4-10FP group compared to the PBS group $(0.4 \%$ vs $3.0 \% ; p=0.029$, figure 1 ).

Synovial inflammation was mild (characteristic of this model) and did not change after intra-articular injections $(0.4$ and -1.3 points out of 18 for clL4-10FP and PBS respectively).
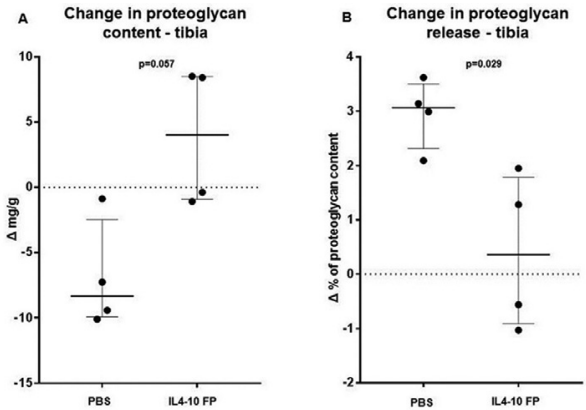

Abstract OP0262 - Figure 1 Chondroprotective effects of clL4-10 fusion protein. (A) change in proteoglycan content between left (control) and right (injected) knees. Values are expressed per animal, representing a mean of 8 samples, and as median \pm IQR. (B) change in release of proteoglycans between left (control) and right (injected) knees. Values are expressed per animal, representing a mean of 8 samples, and as median \pm IQR.

Conclusions: Repetitive intra-articular injection with canine IL4-10FP in dogs did not lead to antibody formation. Dogs treated with clL4-10FP showed improved joint loading compared to PBS treated dogs, reflecting an analgesic effect. Recovery of proteoglycan content and normalisation of release in clL4-10FP treated dogs indicate a chondroprotective effect. Synovial inflammation in the Groove model was too mild to be changed significantly. These results clearly warrant further research to develop IL4-10FP as a DMOAD.

Acknowledgements: This work is supported by the Dutch Arthritis Foundation project NR12-2-202

Disclosure of Interest: None declared

DOI: 10.1136/annrheumdis-2018-eular.1665

\section{OP0263 CLAUDIN-11 REGULATES BONE HOMEOSTASIS VIA BIDIRECTIONAL EPHB4-EPHRINB2 SIGNALLING}

C.-H. Lee ${ }^{1^{*}}$, J.M. Baek², Y.J. Choi ${ }^{3}$, W.-H. Yoo ${ }^{3}$, M.S. Lee ${ }^{4}$, J.-Y. Kim² ${ }^{2}$ ${ }^{1}$ Wonkwang University Hospital; ${ }^{2}$ Anatomy, Wonkwang University, Iksan; ${ }^{3}$ Internal Medicine, Chonbuk National University Hospital, Jeonju; ${ }^{4}$ Internal Medicine, Wonkwang University Hospital, Iksan, Korea, Republic Of

Background: Claudins (Cldns) are well-established components of tight junctions (TJs) that play a pivotal role in the modulation of paracellular permeability Several studies have explored the physiologic aspects of Cldn family members in bone metabolism. However, the effect of Cldn11, a major component of central nervous system myelin, on bone homeostasis has not been reported.

Objectives: This study was performed to identify the effects of Cldn on bone metabolism via regulation of osteoclast and osteoblast differentiation and their function

Methods: We performed various in vitro and in vivo studies using gain- and lossof function of Cldn11 that is belong to the Cldn group. Osteoclast formation from bone marrow cells (BMC) and Osteoblast formation was evaluated in specific condition with over-expression or down- regulation of Cldn11. The expression of osteoclast associated gene and osteoblast related gene mRNA were assessed 